\title{
DEVELOPMENTS IN ENTRY VEHICLE TECHNOLOGY
}

by

\section{ALVIN SEIF F}

Nation al Aeronautics and Space Administration

Moffett Field, California

AIAA Paper

No. 64-528

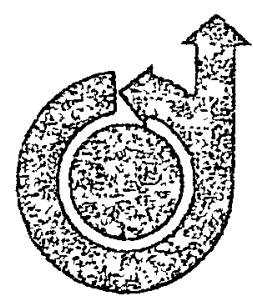

First publication rights reserved by American Institute of Aeronautics and Astronautıcs, 1290 Sixth Avenue, New York. N. Y. 10019 Abstracts may be published without permission if credit is given to author and to AlAA. (Price-AlAA Member 50c, Non-Member \$1.00). 


\title{
DEVEIOPMENTS IN ENTRY VEHIELE TECHNOLOGY
}

\author{
By Alvin Selff
}

Chief, Vehicle Environment Division

NASA, Ames Research Center, Moffett Field, Calif.

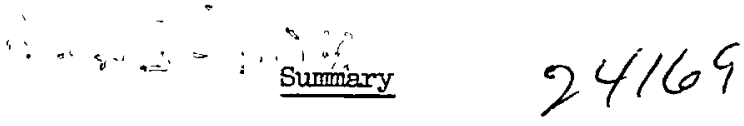

Entry vehicle problems related to Mars entry of an unmanned probe and Earth entry well above the escape speed are discussed, and some recent laboratory research findings related to high-speed entry problems are summarized.

Design for steep entry into some of the proposed Mars atmospheres appears to be infeasible because of the extremely low $\mathrm{m} / \mathrm{C}_{\mathrm{D}} \mathrm{A}$ required for soft landing. Hence, missions to search for life on Mars and to make physical measurements of the martian surface cannot be confidently undertaken until the questions about the atmosphere are resolved. Consideration of an atmospheric sounding probe as the initial Mars entry vehicle is therefore indicated. Such a vehicle need not be a soft lander, but can be a low $\mathrm{m} / \mathrm{CD}_{\mathrm{D}} \mathrm{A}$ capsule which measures the properties of the atmosphere in flight. In the event the minimum pressure atmospheres are confirmed, soft landing may still be achievable by use of extremely shallow entry angles, including as a limit the constant altitude deceleration trajectory. This solution has its attendant problems, chiefly of requiring mach more sophisticated guidance and control than the steep ballistic entry.

For entry into planetary atmospheres, at_speeds - much greater than 40,000 feet per second, use of entry vehicles with all surfaces swept back relative to the stream (such as cones) is indicated to prevent radiative heat transfer from becoming catastrophically large. With optimum sweepback, lifting and nonlifting vehicles on ballistic and shallow angle entry can reject to the atmosphere approximately 99 percent of the entry kinetic energy at speeds up to twice Earth escape speed.

Items selected from recent research are related to cone radiative heating at high speeds, combustion of ablation shields, ablation products radiation, injected gas interaction with the exter. nal flow, and atmospheric gas radiation of nitrogen, carbon-dioxide mixtures.

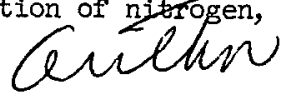

\section{Introduction}

With the entry problems of intercontinental ballistic vehicles and near-Earth satellites now, for the most part, successfully behind them, and with current work apparently yielding needed answers to problems of entry at Earth escape speed, entry vehicle designers and research specialists can look forward to the design of Mars probes and to the problems of Earth entry at speeds up to twice escape speed on return from interplanetary missions. The Mars entry problem is presently complicated by the lack of precise knowledge of the Mars atmosphere. It is generally agreed, however, that the Mars atmosphere has a much lower surface density than that of Earth, and this has a predominant influence on the design of ballistic probes for the Mars atmosphere. The other problem, the major extension of entry speed apparently required for the interplanetary missions, is primarily a problem of heating and heat protection. One wonders how high the entry speed can become before entry bodies suffer the experience of many meteors and become totally vaporized during entry. Not only does the convective heat transfer formule show a dependence on the cube of velocity, but the radiative heating increases in this range as the 15th power of velocity, and becomes predominant. It then becomes necessary to optimize the configuration with respect to total heating to achieve successful entry. ${ }^{1,2}$

The ablation shield is very largely responsible for the success of the entry vehicle efforts to date. Its processes are very complex, and have often been treated by semiempirical methods which have proved adequate for design. Recent laboratory research, however, has shed new light on the performance of these shields, and shows some effects which were largely overlooked until now. The shields made of organic polymers can, and apparently as a rule do, burm in flight. They discharge gases which glow more brightly, under some conditions, than the air whose radiative component is carefully estimated while the other gases are ignored. At high heating rates, they may discharge gas at such a rate as to affect the external flow configurations, and measurably alter the gasdynamic forces - and-moments.

These effects and one other, the high temperature radiative property of gases expected to occur on Mars and Venus, will be briefly discussed in this peper. We will start, however, with some general remarks on the problems of the advanced missions listed in the opening paragraph. The principal problem of the unmanned Mars mission being the low density atmosphere, we will develop its implications for vehicle design. Also, since it is clear that the characteristics of the Mars atmosphere need to be better determined, a means of achieving this will be described. Then the optimum body problem for high-speed entry will be reviewed, and some estimates of the fractions of entry kinetic energy going into body heating shown. The results of an initial evaluation of the problem of tip burnoff on the optimum conical shapes will be presented.

\section{Notation}

a) limit value of acceleration

A frontal area

$A_{S} \quad$ surface area

B dimensionless ballistic coefficient, $\frac{C_{D} \rho_{O} A}{\beta m \sin \theta_{E}}$

$\mathrm{C}_{\mathrm{D}}$ drag coefficient

$\mathrm{C}_{\mathrm{H}}$ heat-transfer coefficient, $\frac{\mathrm{dQ} / \mathrm{dt}}{(1 / 2) \rho V^{3} \mathrm{~A}}$

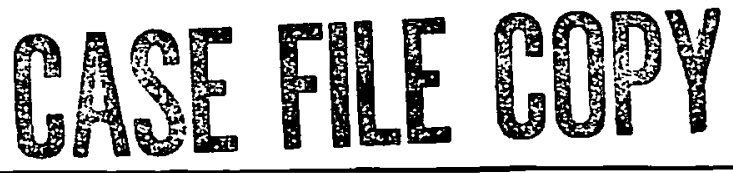



$\mathrm{c}_{\mathrm{m}_{\mathrm{x}_{i}}} \underset{\text { initial slope of static pitching-moment }}{\text { curve }}$

$\mathrm{CN}_{\alpha_{i}}$ initial slope of normal force curve

a body diameter

E power radiated per unit volume

g acceleration due to gravity.

h altitude

$h_{t} \quad$ total enthalpy

H scale height, $\frac{\mathrm{RT}}{\mathrm{g}}$

$\frac{\mathrm{L}}{\mathrm{D}} \quad$ lift-drag ratio

m vehicle mass

m rate of mass injection or ablation

M Mach number

p

$p_{t} \quad$ total pressure at stagnation point

$\dot{q}_{a} \quad$ heating rate due to ablation product radiation

$\dot{q}_{0} \quad$ heating rate at zero ablation

Q total heat input on trajectory

$r_{b} \quad$ body radius of cross section

$r_{n} \quad$ tip radius

$\mathrm{R} \quad$ body nose radius

$\mathrm{Re}$, limit value of Reynolds number based on local flow properties

average weight of structure and heat shield per unit surface area

T

$\mathrm{U}$

$\mathrm{V}$

W

$\beta$

$\rho$

$\bar{\rho} \quad \frac{\rho}{\rho_{0}}$

$\theta$

$\theta_{c} \quad$ cone half-angle

$\zeta \quad$ energy to melt and vaporize unit mass of ablator
Subscripts

$\begin{array}{ll}\text { b } & \text { body } \\ \text { e } & \text { boundary-layer edge } \\ \text { E } & \text { entry } \\ \text { p } & \text { parachute deployment } \\ \text { s } & \text { satellite } \\ \text { S+HS } & \text { structure plus heat shield } \\ \text { strat } & \text { stratosphere } \\ t & \text { terminal } \\ \text { W } & \text { body surface } \\ 0 & \text { zero altitude } \\ \text { a } & \text { behind bow shock wave } \\ \text { max } & \text { maximum } \\ \infty & \text { free stream }\end{array}$

\section{Mars Probe Design}

Best current estimates are that the ground level pressure at Mars is in the range of 1 to 2.5 percent of Earth sea-level pressure, and therefore corresponds to pressure in the Earth's atmosphere at altitudes between 80,000 and 100,000 feet. Since proposed probe missions generally require the soft landing of an instrument package on the planet surface, the vehicle mist be designed to decelerate to nearly zero speed in the low density atmosphere. Now the equation governing the deceleration to low speed on ballistic entry is, to a very good order of approximation, the equation of Allen and Eggers, ${ }^{3}$

$\frac{\mathrm{V}}{\mathrm{V}_{\mathrm{E}}}=e^{-\frac{\mathrm{B}}{2} \bar{\rho}}=e^{-\frac{\mathrm{C}_{D} \rho \mathrm{A}}{\beta \mathrm{m} \sin \theta_{E}}}=e^{-\frac{\mathrm{C}_{D} \mathrm{~A}}{\mathrm{~m}} \frac{\mathrm{p}}{\mathrm{g} \sin \theta_{E}}}$

which relates the value of $m / C D A$ to the velocity ratio, $v / V_{E}$, required at a given level of atmospheric density or pressure. It is well known that equation ( 1 ) does not hold during the final stages of descent, in which the neglected weight force becomes comparable to drag force. There, the vehicle may approach terminal velocity, the velocity at which weight force equals drag force,

$$
v_{t}=\sqrt{\frac{2}{\rho} \frac{W}{C_{D A}}}
$$

It can easily be calculated that the terminal velocities obtained in the Mars atmosphere with values of $m / C_{D} A$, usually considered are much too high for soft landing. Hence, a parachute or other retarding device is required. The parachute, like other drag generating elements, loses effectiveness in the low density atmosphere, and for minimm system weight must be deployed at a rather high altitude. Equation ( 1 ) is then applicable to calculating the value of $\mathrm{m} / \mathrm{CDA}$ needed to bring the probe to parachute deployment velocity at the specified altitude, $h_{p}$. The results of such a calculation are shown in Fig. I in terms of the presentiy uncertain atmospheric parameters $p_{o}$ and $H$, where $H$, the scale 
height, is equal to $1 / \beta$. Parachute deployment is assumed to occur at $0.05 \mathrm{~V}_{\mathrm{E}}$, corresponding to about 1,250 ft/sec for typical Mars entry velocities, and at 30,000 or 50,000 feet altitude. The value of $\mathrm{m} / \mathrm{CDA}$ required is presented as a function of $\mathrm{p}_{\mathrm{O}} / \sin \theta_{\mathrm{E}}$. For vertical entry, the abscissa is the ground level atmospheric pressure and may be correlated with the tick marks indicating the G, I, and $J$ model atmospheres of the Jet Propulsion Iraboratory.

Some very low values of $\mathrm{m} / \mathrm{CDA}$ are required with some of the assumed atmospheres. For example, on vertical entry with parachute deployment at 50,000 feet, $p_{0}$ of 10 millibars, and $H$ of 25,600 feet, $\mathrm{m} / \mathrm{CDA}_{\mathrm{DA}}$ is $0.02 \mathrm{slug} / \mathrm{ft}^{2}$. Furthermore, the variation in the design value of $\mathrm{m} / \mathrm{C}_{\mathrm{D}} \mathrm{A}$ corresponding to the surface pressure range of 10 to 40 millibars and the scale height range indicated is greater than an order of magnitude. This is perhaps the greatest effect of current Mars atmosphere uncertainties on entry probe design.*

Now to appreciate what is represented by these very low values of $\mathrm{m} / \mathrm{C}_{\mathrm{D} A}$, consider the corresponding vehicle densities presented in Fig. 2. The vehicle bulk density, total weight at Earth divided by total volume, is plotted as a function of frontal radius for spherical bodies. Note that the scale maximum is less than one-tenth of the density of water, and the smallest value plotted is onethousandth the density of water. The significance of this is clear. The vehicle is largely empty, and is itself little more than a balloon deceleration device. For the middle curve on this figure, $\mathrm{m} / \mathrm{CDA}=0.1$, the total weight for-all-radii-is-that of an aluminum skin of 0.05 -inch thickness, with no weight remaining for payload or other purpose. For the lower curve, the total-weight aluminum skin thickness becomes 0.01 inch. It can safely be concluded that the design of such vehicles is no more than marginally possible.

This fact is further amplified in Fig. 3, where the weight available for structure and heat shield is expressed in pounds per square foot of surface for perhaps the most efficient shape, a flat-faced vehicle like the Apollo. A 100-pound payload is considered. For a specified value of $\mathrm{m} / \mathrm{CDA}_{\mathrm{A}}$, both the vehicle weight and the surface area increase directly with the frontal area. Increasing the vehicle radius therefore results in an asymptotic approach to limiting values of $s$ as shown. For $\mathrm{m} / \mathrm{CDA}$ of $0.5 \mathrm{slug} / \mathrm{ft}^{2}$, the asymptotic value $8 \mathrm{lb} / \mathrm{ft}^{2}$ available for structure and heat shield is well within present design capability. The asyroptotic velue diminishes directly with $\mathrm{m} / \mathrm{C}_{\mathrm{D}} \mathrm{A}$, becoming, for example, $1.6 \mathrm{Ib} / \mathrm{ft}^{2}$ for $\mathrm{m} / \mathrm{C}_{\mathrm{D}} \mathrm{A}$ of 0.1 , and $0.32 \mathrm{lb} / \mathrm{ft}^{2}$ for $\mathrm{m} / \mathrm{CD}_{\mathrm{A}}$ of 0.02 . One can imagine a boundary line extending across the lower part of this figure, separating attainable from unattainable structures. This line, presently ill defined, slopes upward to the right since vehicle rigidity diminishes with increasing size.

It might be gathered from the above that the design of a soft-landing ballistic-entry probe for Mars is marginal if not impossible within the present uncertain definition of the Mars atmosphere,

*The results shown in Fig. 1 are applicable to other values of scale height and parachute deployment altitude, as long as the ratios $h_{p} / H$ remain the same. since, as in all design problems, the design must cope with the worst conditions to be expected. Two lines of action should result from this conclusion: One is the study of light-weight structural concepts, including unconventional concepts. Pressurlzed structures may play an important role, for example, just as they have for rocket boosters. Second, we should find out more about the Mars atmosphere to reduce the uncertainty and eliminate the necessity to design for atmospheric conditions worse than may actualiy exist.

If the surface pressure of the Mars atmosphere is as low as the lowest of the current models, the question then raised is whether a probe vehicle of useful density can in any way be soft landed by gasdynamic braking. Equation ( 1 ) shows that $m / C_{D A}$ may be increased for small values of $\theta_{\mathrm{E}}$. In the limit, $\theta_{\mathrm{E}}=0$, there is the possibility of dissipating the vehicle energy in horizontal flight.

Such constant altitude deceleration trajectorles were discussed in reference 2 for manned vehicles and can, in principle, be applied to unmanned probes as well. The controlled use of lift is required to maintain this trajectory, but for Mars, the values of $L / D$ are very small (less than 0.1 ) at speeds up to 2.5 times circular.

The altitude selection is to a degree arbitrary, since there is no absolute acceleration limit for an unmanned payload, and Reynolds numbers tend to be below values for turbulent boundary layer. Very high altitudes, for example, above 100,000 feet, can be selected to be sure of ground clearance and to-al-low-room-for final-descent-by-parachute although high altitudes lead to greater total heat input than low altitudes, if laminar. boundary layer is obtained at both. The minimum velocity to which circular flight paths can be maintained depends on the altitude selected, $\mathrm{m} / \mathrm{C}_{\mathrm{D} A}$, and $(\mathrm{I} / \mathrm{D})_{\max }$, but is generally a small fraction of satellite velocity. Below this speed, a shallow-angle descent is followed to the speed of parachute deployment. Mars entry in terms of such a trajectory appears feasible for the lowest pressure atmosphere now proposed. However, this solution is not without penalty, since the guidance and control problems are mach increased for this flight path compared to simple ballistic entry.

In terms of the above discussions, the need for data on the density structure of the Mars atmosphere is clear. The question then posed is how to obtain data of the required accuracy. Although remote measurements from a spacecraft flying by the planet may provide it, the most direct method of measuring the atmosphere is by use of an entry probe. From the arguments already given, it is evident that this atmosphere sounding probe cannot be dependent on soft landing for its function. However, as has been discussed in reference 4 , it is possible to measure the atmospheric properties while the probe is in hypersonic flight. 
If the vehicle does not soft land, then it must comminicate all 1ts data prior to landing. For $a$ blunt vehicle, ${ }^{*}$ there will be a period of cormunication blackout, so that the period available for communicating the trajectory data begins after blackout and ends at surface impact. This time must not be allowed to become too short, and the means of protracting it is, again, the reduction of $\mathrm{m} / \mathrm{C}_{\mathrm{DA}}$. This is illustrated by Fig. 4. For two atmospheres, one of which is conpletely isothermal and the other of which has a falling temperature up to a tropopause, like the Earth's, there is shown the available commication time as a function of $\mathrm{m} / \mathrm{CDA}_{\mathrm{DA}}$ and surface pressure. These results are for the most stringent case, vertical entry, and they indicate that for 10 millibars surface pressure, communications time in excess of 15 seconds will be obtained for a vehicle $\mathrm{m} / \mathrm{C}_{\mathrm{D}} \mathrm{A}$ of $0.2 \mathrm{slug} / \mathrm{ft}^{2}$. Comminications blackout has been assumed to end at $10,000 \mathrm{ft} / \mathrm{sec}$ in these calculations. In 15 seconds with a commuications rate of 100 bits per second, such as might be provided by relay link to the parent spacecraft, the equivalent of 250 mumbers of 2 -percent accuracy can be comminicated. Hence, such a communication period is compatible with sending a useful amount of data.

Measurements to be made with such a vehicle include (Fig. 5): deceleration, which provides a means for deducing atmospheric density and tracking of the vehicle velocity; static and pitot pressure, which, at the time of impact, define the surface pressure and terminal velocity; atmospheric temperature, which is measurable in low-speed flight during terminal descent; and certain elements of the shocklayer emission spectrum, which can be used to identify atmospheric gases and their concentrations. Since detailed discussion of these measurements is not appropriate here, reference 4 will be augmented by a report now in preparation.

Vehicle parameters which appear suitable for use in such a probe are indicated in Fig. 6 . The intent here is a minimum weight device which can be considered as one experiment of several carried by the spacecraft. (Others could, in fact, be entry vehicles differing in instruments and design, to increase the probability of some successful measurement of the atmospheric structure.) The weight of the instruments and electronics including data storage and conmunications equipment has been estimated as being as little as 12 pounds. For an $\mathrm{m} / \mathrm{CDA}_{\mathrm{D}}$ of $0.25 \mathrm{slug} / \mathrm{ft}^{2}$, weights corresponding to radii of 12 and 15 inches are 23.7 and 37 pounds. Thus, a reasonable allowance for structural and heat shield weight is available in these sizes. The capsule volume is largely empty, even for the l-foot

*The use of a slender vehicle to avoid communications blackout has been proposed. However, the slender vehicle can maintain communications only when its angle of attack is of the order of a few degrees or less, because at larger angles of attack it is no longer "slender." "Since this is difficult to insure on a vehicle which/is -launched remotely and under difficult conditions, the possibility that no data will be obtained must be considered. In addition, the slender vehicle does not lend itseif to the use of accelerometer measurements except at very low altitudes. Since it does not decelerate appreciably, the time of transit of the slender vehicle through the atmosphere, from 250,000 feet altitude to impact, is of the order of 10 seconds. This is the total period for data acquisition and transmittal. radius, consistent with earlier conclusions. The choice of a spherical configuration permits more exact treatment of angle-of-attack effects within the limitations of the total data quantity imposed by communications. (The advantages of spherical geometry were pointed out by Victor Peterson at Ames Research Center.) Work on this atmosphere sounding probe concept is contimuing at Ames Research Center.

\section{Earth Eintry at Up to Twice the Escape Speed}

Turning now to the topic of very high-speed entry vehicles, such as will be required on return from interplanetary missions or entry into some of the planets, we note that the predominant heat transfer to blunt vehicles is the intense radiation from hot gases in the shock layer. The speed dependence of this radiation is such that in the velocity range from satellite speed to twice satellite speed, an order of magnitude increase accompanies a velocity increase of $1.5 \mathrm{~km} / \mathrm{sec}$. A summary of theoretical data for normal shock waves in air is presented in Fig. 7. The Iuminous power per unit volume of gas ranges over 11 orders of magnitude as the velocity ranges from 1 to 4 times satellite speed for the altitude range from 20 to $70 \mathrm{kilo}$ meters. Note that straight line segments can be used to represent these data very adequately. The radiant power varies as $U^{15.45}$ at speeds from 8 to $13.7 \mathrm{~km} / \mathrm{sec}$ and as $U^{5.05}$ at higher speeds. 1 The theoretical curves are experimentally confirmed* within a factor of 2 out to a velocity of $13 \mathrm{~km} / \mathrm{sec}$, but are unconfirmed at higher speeds.

Above the escape speed from Earth, the radiative heating to blunt ballistic entry vehicles becomes prohibitive. Relief from this heating is afforded by sweeping back the bow shock wave, $1,2,8,7$ since the temperature behind a shock wave is a function of the component of velocity normal to the wave. The effect of sweepback on total heat transfer, sum of convective and radiative, has now been calculated for three classes of trajectories, ballistic, 1 a constant altitude deceleration, and $a$ constant Reynolds number descent. Preliminary results for the shallow angle trajectories were given in reference 2 . Other unpublished resilts have since been obtained by my colleague Michael Tauber and myself. Representative results are show in Fig. 8, for an entry velocity of 2.4 times near-Earth satellite velocity. Note that the total heat $Q$ entering the body on the trajectory is, for optimum cone angles, less than 1 percent of the entry kinetic energy, but for higher and lower cone angles, it cen become much larger. The slope of the curves at $45^{\circ}$ cone angle indicates the severity of the heating problem of the unswept blunt body.

For all three classes of trajectories, the optirmm cone angle is between $25^{\circ}$ and $35^{\circ}$. That

*The available experimental measurements do not include the vacuum ultraviolet region of the spectrum, but have been compared with theory in the visible and infrared. In a recent paper, Breene ${ }^{5}$ has, recomputed the power in the wavelength region from 800 to $1100 \AA$ generated by the deionization reaction of $\mathbb{N}^{+}$, and obtained an appreciably higher value than earlier calculations. While all of the theoretical values shown in Fig. 7 include this reaction, there is some uncertainty about the magnitude of its contribution to the radiation. 
this optimum is not the same for every trajectory is are to the differences in velocity-density relationship. For the ballistic trajectory, for example, the maximum radiative input occurs at

$V=0.9 V_{E}$, while for the constant altitude deceleration, the radiation is maximum at $V=V_{E}$. This tends to increase the importance of radiative transfer for the constant altitude trajectory and to drive the optimum to smaller cone angles.

It can be shown from the heating equations that the constant Reynolds number trajectory is an optimum or near optimim trajectory. The numerical results bear this out by showing the least value of total heating of the three classes considered. At optimum, the total heating is in all cases predominantly convective ( 85 percent or more of the total).

All of these trajectories have been selected to restrict the Reynolds number below a specified limit to remain in the flight regime where laminar boundary layers may occur. Now the correct value for this limit is not well established and may, in fact, depend on some particular choices such as the choice of heat shield material. Based on available experience, the limit value of 10 million has been applied in these examples, but changes in this value can easily be inserted in the analysis. The constantaltitude trajectory is also restricted to a $10 \mathrm{~g}$ acceleration limit, and it is noteworthy that with large entry bodies both the acceleration and Reynolds number limits can be met while still obtaining nearly the same heat input energy fractions as in ballistic entry where the accelerations are far beyond muan tolerance. The total heat input curve for the constant-altitude trajectory is discontinuous at the optimim point, which marks the intersection of the Reynolds number limited curve (to the left) and the acceleration linited curve (to the right). The optimim point always occurs at this intersection, that is, at a point where both the acceleration and Reymolds number limits are reached.

It is a feature of the results that the optimum bodies are always near $30^{\circ}$ cone half-angle. The angle tends to diminish with increasing speed, but, up to three times satellite velocity, a $30^{\circ}$ cone could be used with little penalty. Bodies of this near optinum geometry are shown in Fig. 9. The ballistic body is the complete cone. Since lift is required on the shallow angle trajectories, lifting configurations generating $L / D$ of 0.5 which might be used for these cases are also shown. The lifting configuration is formed by cutting off the cone asymetrically, at the left by the method of Eggers ${ }^{8}$ (removing part of the cone upper surface with a plane cut through the apex) and at the right by the method of Shapland ${ }^{9}$ (base plane skewed relative to the cone axis).

The critical question which cannot be ignored is the degree of tip burnoff which will occur with these pointed entry vehicles. Allen has suggested the use of a cusped tip or a rod fed through the tip to combat tip burnoff. However, until now, no calculations had been made to determine the extent to which tips of the best available ablation miterials would be ablated on entry trajectories. My colleague Michael Tauber has programed our heating equations for the tip and solved them numerically on the IBM 7094 computer. His results are shown in Fig. 10 for constant altitude deceleration. (Results for ballistic entry, not shown, are even more favorable.) For entry speeds up to 3 times satellite velocity, the tip radius would remain less than 10 percent of the base radius for a graphite tip, according to these calculations. A vaporizing quartz tip (no liquid runoff) is nearly as good, while a Teflon tip could not be used above about $\mathrm{V}_{\mathrm{E}} / \mathrm{V}_{\mathrm{S}}$ of 1.8. These calculations and these materials require further study and experimental confirmation.

The calculated results, however, are encouraging with respect to the prospects for retaining highly swept bow waves over most of the vehicle surface. It is to be noted that rounding of just the tip.will reduce convective heating over most of the vehicle while subjecting the tip to increased radiative heating.

\section{Other Topics From Current Research}

Some preliminary work aimed at proviaing experimental confirmation of sweepback theory for computing shock layer radiation on cones was reported. 6 This work has now been completed by Compton and Cooper, 10 and is shown in Fig. 11. Total radiation from the shock layer of a $30^{\circ}$ half angle cone is plotted as a function of equilibrium air density in the shock layer. The interpretation of the data has been complicated by the occurrence of two effects: (1) radiation from the products of ablation comparable in magnitude to the air radiation, and (2) sizable contributions of nonequilibrium radiation over most of the density range of the experiments. These contributions are apparent in the data. The ablation products radiation was studied experimentally by use of models of-differ = ent materials, including, for limited conditions, nonablating metel models. With polyethylene models (circular symbols), the measured radiation was from 1.5 to 3 times that expected from the air. With the nonablating metal models (square symbols), and the polyformaldehyde models (triangular symbols), radiation in agreement with the predicted total of equilibrium plus nonequilibrium air radiation was measured in the visible spectrum.

The nonequilibrium radiative contribution was estimated by Compton using a sweepback theory to convert results from low-speed normal shock waves to. conical flow conditions. His procedure is apparently reasonably successful in predicting the total air radiation. One can conclude that the data support sweepback theory when allowance is made for ablation product radiation and nonequilibrium air rediation.

For flight conditions, analyses ${ }^{1,2}$ have shown that nonequilibrium radiation contributes negligibly to the heating. It is likely true,-however, that ablation products radiation will, for some materials, remain comparable to or greater than air radiation for optimum cones in flight. Attention has recently been given to predicting ablation product radiation on cones. 11

A subject which has received prominent attention in the last two years because of its importance for planetary entry is radiation from gas mixtures of nitrogen and carbon dioxide, believed to occur on both Mars and Venus. Cyanogen radiation in the vicinity of $3883 \AA$ is very intense in these mixtures and at moderate flight speeds increases the radiative heating considerably above 
that of air. James Arnold of Ames Research Center has been acquiring new data by free-flight techniques in the speed range around $30,000 \mathrm{ft} / \mathrm{sec}$, and has prepared a correlation plot to show the interrelation of all the measurements which have now been published 12,13 for mixtures containing 7.5 to 9 percent $\mathrm{CO}_{2}$, reproduced here in Fig. 12. At the lower speeds, the $\mathrm{CO}_{2}$ mixtures generate between 1 and 2 orders of magnitude more radiation than does air, for equal gas density. As the speed is increased and the cyanogen dissociates, the gas mixture radiation approaches that of air, and above $10 \mathrm{~km} / \mathrm{sec}$ is comparable to air radiation or perhaps slightly 'below. The two theoretical lines in this flgure are by different authors (solid line, Spiegel; dashed line, Breene), and are thus more representative of different theoretical assumptions than of different gas compositions. Experimentally, the effect of changing from 7-1/2- to 9-percent $\mathrm{CO}_{2}$ is small (see data of James). The way in which the free-flight data correlates with the shock-tube data ${ }^{13}$ in this figure is very satisfactory.

The relatively level region in the $\mathrm{CO}_{2}$ mixture data between 6.5 and $9.5 \mathrm{~km} / \mathrm{sec}$ has some interesting and somewhat important implications. One pointed out by Arnold pertains to the distribution of radiative intensity behind a curved shock wave. The radiation will not fall off as rapidly w1th distance from the stagnation point in the $\mathrm{CO}_{2}$ mixture as in air, because the radiation is nearly independent of speed in this region. Also, it is clear that in choosing optimum cones to minimize radiation, somewhat smaller angles will be required in these mixtures than in air. An example of this for Mars entry has been given in reference 2.

For blunt as well as conical bodies, radiation from ablation products can be a source of considerable radiative heating of the vehicle. One of the materials which has been studied in the laboratory for which this is very pronounced is the polycarbonate, Lexan;* laboratory data ${ }^{14}$ showing the radiative heating of models by the ablation products are shown in Fig. 13. Analysis of the data has shown that the radiative heating is proportional to the ablation rate, $\dot{m}$, and to the boundary-layer edge temperature $\mathrm{T}_{\mathrm{e}} \mathrm{3.5}^{\mathrm{s}}$, both of which dependences are intuitively appealing. The data shown were obtained at velocities from 4.5 to $7.6 \mathrm{~km} / \mathrm{sec}$ and at ambient pressures from 0.02 (c1rcles) to 0.2 atmosphere (diamonds). A systematic effect of the level of pressure or density found to be present can be correlated for this range by the factor $\left(\rho_{e} / \rho_{0}\right)^{0.10}$ which is incorporated in the ordinate. The influence of the ambient density is to change the equilibrium composition of the products of ablation and the rate of spproach to equilibrium. The dependence on body radius shown on the abscissa was analyt1cally derived by Craig and Davy, and is critically dependent on the assumption that the profiles normal to the surface of sblation products concentrations and of velocity remain similer and of corresponding thickness with change in body radius. All the data shown here were obtained with small models

$(\mathrm{R}=0.508 \mathrm{~cm})$, and no verification of the linear dependence on $R$ is available. The significance of this predicted dependence is that large vehicles tend to have large values of the abscissa and hence large ablation products heating. Further work is required to verify or replace these scaling rules.

*Results obtained with this material vary greatly from batch to batch. Differences in molecular weight and perhaps other chemical differences cause some lots of material to radiate much more brightly than others.
A fact brought home by these investigations has been the posstble importance of choosing ablation shield materials whose products do not radiate strongly. The polycarbonate material is the most intense radiator which has been found. Other materials show lower intensities by an order of magnitude or more. William Borucki at Ames Research Center has been studying the spectra of the ablation products radiation to identify principal radiators. His preliminary results indicate that cyanogen and $C_{2}$ Swan bands occur prominently with Lexan and polyethylene ablators. The cyanogen is produced by reaction of carbon from the ablator with nitrogen in the air. The equilibrium and rate of approach to equilibrium of this reaction is thus an important determinant of the amount of radiation produced. It is also possible that small solid particles are discharged or formed in the boundary layer and radiate as epproximately black bodies.

Chemical interaction between heat-shield materials and the air or other atmosphere has also been measured in studies of the behavior of heat shields in various atmospheres. While the possibility of heat-shield combustion has long been recognized, particularly in relation to carbon shields, the early analysis and application of heat shields has generally ignored this effect. The degree to which it can affect heat-shield performance is shown in Fig. 14 for a phenolic nylon shield in laboratory tests. 15 Tested at identical stream conditions in nitrogen and air, the heat shield assumed temperatures as much as $400^{\circ} \mathrm{F}$ greater in the air, and suffered 60 percent more mass loss. Nick Vojvodich has analyzed data of this kind for a range of conditions and concludes that chemical reactions are generating heat quantities equal to or greater than the expected convective transfer. He further finds that ebout $1 / 3$ of this occurs from combustion of the solid char, while $2 / 3$ occurs in gas-phase reactions. Char loss was experienced in nitrogen and in argon as well as in air, but at 30 to 40 percent the rate experienced in air. Later experiments by John Lundell have shown that char loss also occurs under purely radiative heating of the shield in vacuum. Although char loss under these conditions may be due to spallation, John Parker has suggested that it may also result from chemical consumption of the char by ablation pyrol. ysis gases percolating up through it.

A relationship quite clearly exists between the problem areas treated in the last two figures. of the additional heating caused by ablation products chemical reactions, some part will be by radiative transfer. Theoretical studies have shown ${ }^{18}$ that chemical reactions to be expected include the formation of $\mathrm{CO}$ and $\mathrm{CN}$ by interaction of atmospheric gases with carbon in organic shield materials. These processes are exothermic and, in the case of $\mathrm{CN}$, yield a product of high radiative output. These studies are concerned with effects which are of first-order importance for heat shields, but which have been neglected till now. That we have been able to successfully design shields without understanding these factors can only be attributed to the conservatism of the designs. These effects will be expected to lead to differences in heat-shield effectiveness for atmospheres of differing composition.

There are thus a number of important ways in which ablation shields can affect heating, other than by their intended mechanism of heat absorption 
and blockage. Still another major effect has now been demonstrated by Syvertson and McDevitt.17,18 It is the first contribution in a field that may be called interaction between ablation and external gasdynemics. Shown in Fig. 15 are their data on the initial static pitching stability and normal force of a slender cone with transpiration of gas through the rear 82 percent of the surface length. Firstorder effects on the initial slopes of the stability and normal force curves are observed, for mass injection rates of the order of a few percent of the free-stream mass flow intercepted by the cone base. These are attributed by Syvertson to the formation of a low-energy gas layer over the surface of the cone, consisting of injected gas and having about the same effect as a separated flow layer. It provides a path for equalization of pressure between the windward and sheltered meridians.

For an ablating cone, the variable on the abscissa can be related to the heat-transfer rate. Using Allen's heat-transfer coefficient, $C_{H}$, which is the fraction of the stream kinetic energy converted to heat, ${ }^{1}$ we can show that

$$
\frac{\dot{m}}{\rho_{\infty} V_{\infty} A}=C_{H} \frac{V_{\infty}^{2} / 2}{\zeta}
$$

where $\zeta$ is the energy per unit mass to melt and vaporize the ablation material (expressed in mechanical rather than thermal energy units). This relation shows the importance of velocity in producing this effect. A cone of the geometry tested, in flight at 20,000 ft/sec and 100,000 ft altitude, attains values of the abscissa around 0.01 and 0.04 for-laminar and turbulent boundary-layers, respectively, depending on the value of $\zeta$ assumed and other particulars. Thus the effect is of real significance for slender vehicles. Its importance for vehicles of lower fineness ratio is not yet known.

It has been pointed out that the dynamics of an entry vehicle can be affected by the changes in static properties shown in Fig. 15. Thus, just as changes in dynamic pressure with altitude on ballis. tic entry affect the spring stiffness of the pitching oscillation and produce apparent damping and divergence, variation in the static pitching stability coefficient along the trajectory resulting from changes in $\mathrm{C}_{\mathrm{H}}$ and $\mathrm{V}$ can affect the dynamic convergence of oscillations. Syvertson and McDevitt have discussed these effects. 18

\section{Concluding Remarks}

The forefront of the field of entry vehicle design now lies in the area of design for interplanetary missions, including return from those missions. For Mars entry, the principal problem is that the atmosphere is unknown to a degree that leaves the ballistic parameter of soft-landing vehicles uncertain by an order of magnitude. For the lower limits of atmosphere density, design of steep entering ballistic probes is either marginally possible or impossible. Shallow angle lifting entry can probably be achieved with the lowest pressure atmosphere now envisioned, if the stringent requirements on guidance and control can be met. The necessary first step in the exploration of Mars is to measure the atmospheric density structure, which seems to require sending in a probe vehicle.
Return to Earth from planetary missions at speeds up to twice the escape speed can probably be achieved with pure aerodymamle braking, thereby saving the considerable weight penalty associated with rocket braking. Unlike the situation at lower speeds, successful entry at these high speeds requires the use of optimum bodies, which are approximated by pointed cones of about $30^{\circ}$ half angle. The penalties of departure from near the optimum can be very severe. Based on calculations, it appears possible that by use of appropriate ablation materials, the cone tips can be kept sufficiently sharp throughout entry to keep the radiative heating low over most of the body.

It has now been observed that the ablation shield used to protect the entry body from heating can, depending on the materials used, also contribute to the heating, either by radiation from the ablation products or by exothermic reactions with the atmospheric gases. Furthermore, in cases of high ablation rate, the gas flow from the surface can alter the external flow gasdynamics sufficiently that the forces and moments on the entry body are affected. These observations add to the understanding of entry body processes and study of these effects should improve our capability for designing future systems.

\section{References}

1. Allen, H. J., Seiff, A., and Winovich, W.: Aerodjnamic Heating of Conical Entry Vehicles at Speeds in Excess of Earth Parabolic Speed. NASA TR R-185, 1963.

2. Seiff, A.: Atmosphere Entry Problems of Manned Interplanetary Flight. Presented at AIAA Conference on Engineering Problems of Manned Interplanetary Exploration; Palo Alto, Calif., Oct. 1963.

3. Allen, H. J., and Eggers, A. J.: A Study of the Motion and Aerodynamic Heating of Ballistic Misiles Entering the Earth's Atmosphere at High Supersonic Speeçs. NACA Rep. 1381, 1958.

4. Seiff, A.: Some Possibilities for Determining the Characteristics of the Atmospheres of Mars and Venus From Gas-Dymemic Behavior of a Probe Vehicle. NASA IN D-1770, 1963.

5. Breene, R. G., Nardone, M. C., Zeldin, S. S., and Riethof, T. R.: Radiance of Species in High Temperature Air. G. E. Infor. Rep. R63SD3, 1963.

6. Seiff, A., Goodwin, G., and Wick, B.: H.: Effect of Vehlcle Configuration on Combined Heating Loads With Special Reference to Radiative Heating. NASA-Industry Apollo Technical Conference, Washington, D. C., Juiy 1961.

7. Allen, H. J.: Gas Dynamics Problems of Space Vehicles. NASA SP-24, 1962, pp. 1-19.

3. Eggers, A. J., Jr.: Some Considerations of Ai.craft Configurations Suitable for Long-Range Hypersonic Flight. Presented to the Colston Research Society Symposium on Hypersonic Flow, Un1v. of Bristol, England, April 1959. 
9. Shapland, D. J.: Preliminary Design of a MarsMission Earth Reentry Module. Lockheed Missiles and Space Co. Rep. 4-57-64-3, March 1964.

10. Compton, D. L., and Cooper, D. M.: Measurements of Radiative Heating on Sharp Cones. Paper presented at First Annual Meeting of AIAA, Washington, D. C., June 28 - July 2, 1964. NASA TM X-54,038.

11. Davy, W. C., Craig, R. A., Chapman, G. T., and Compton, D. L.: Ablation Product Radiation From Cones. AIAA Preprint 64-71, 1964.

12. James, C. S.: Experimental Study of Radiative Transport From Hot Gases Simulating in Composition the Atmospheres of Mars and Venus. Paper presented at AIAA/MIT Conference on Physics of Entry into Planetary Atmospheres, Alig. 1963. AIAA Paper 63-455.

13. Gruszczynski, J. S., and Warren, W. R.: Experimental Heat Transfer Studies of Hypervelocity Flight in Planetary Atmospheres. AIAA Paper 63-450, 1963.
14. Craig, R. A., and Davy, W. C.: Thermal Radiation From Ablation Products Injected Into a Hypersonic Shock Layer. NASA TN D-1978, 1963.

15. Vojvodich, N. S., and Pope, R. B.: Effect of Gas Composition on the Ablation Behavior of a Charring Material. AIAA Jour., March 1964, pp. 536-542.

16. Kratsch, K. M., Hearne, L. F., and McChesney, H. R.: Theory for the Thermophysical Performance of Charring Organic HeatShield Composites. Lockheed Missiles and Space Co. Rep. 803099, 2-60-63-7, Oct. 1963.

17. Syvertson, C. A., and McDevitt, J. B.: Effects of Mass Addition on the Stability of Slender Cones at Hypersonic Speeds. AIAA Jour., April 1963, pp. 939-940.

18. Syvertson, C. A., and McDevitt, J. B.: Effects of Mass Transfer Cooling on the Static Stability and Dynamic Motions of Slender Entry Vehicles. Paper presented at Eighth Symp. on Ballistic Missile and Space Technology, San Diego, Calif., Oct. 15-18. 1963.

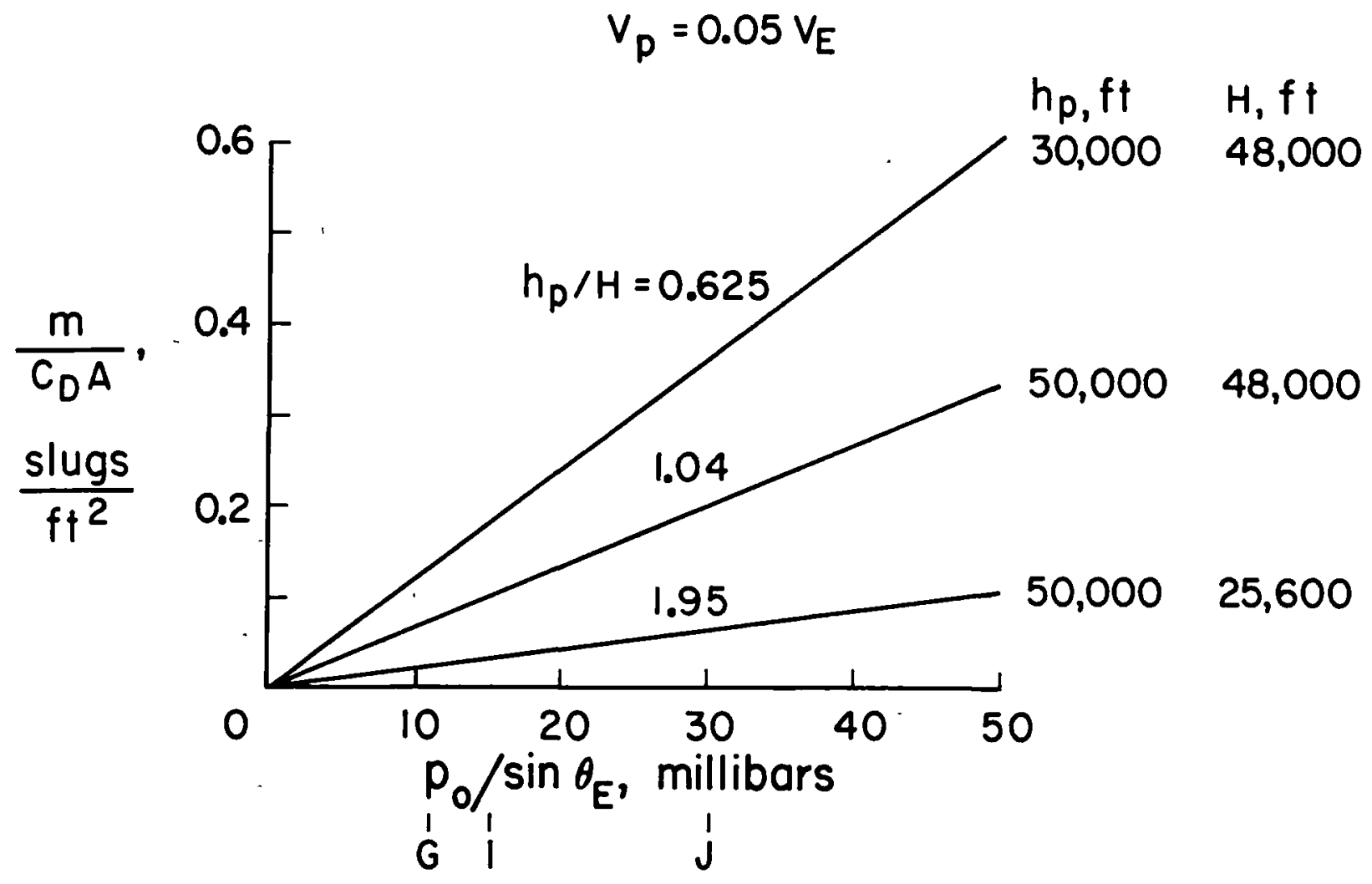

Fig. 1. $-m / C_{D} A$ requirements for soft landing. 

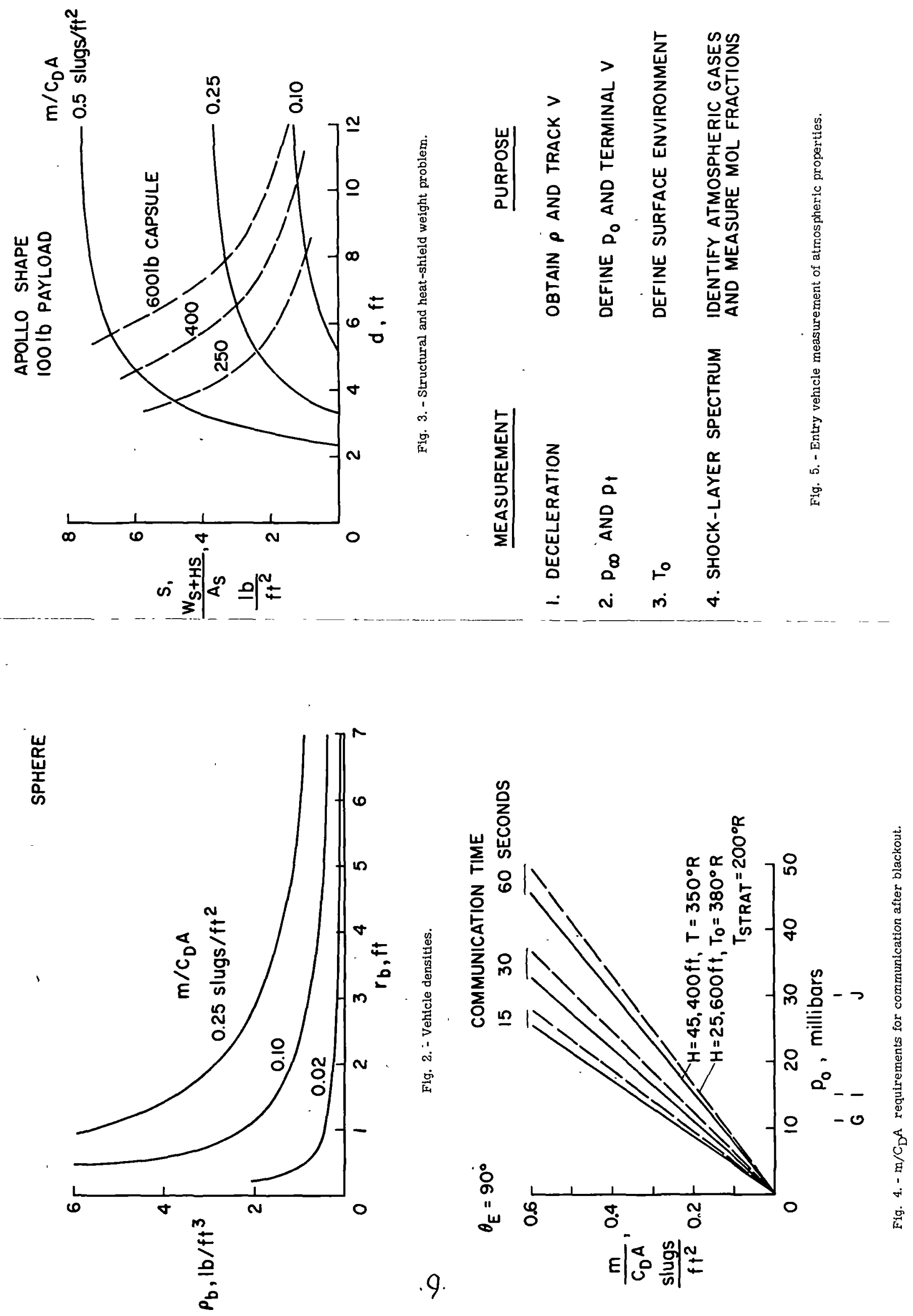

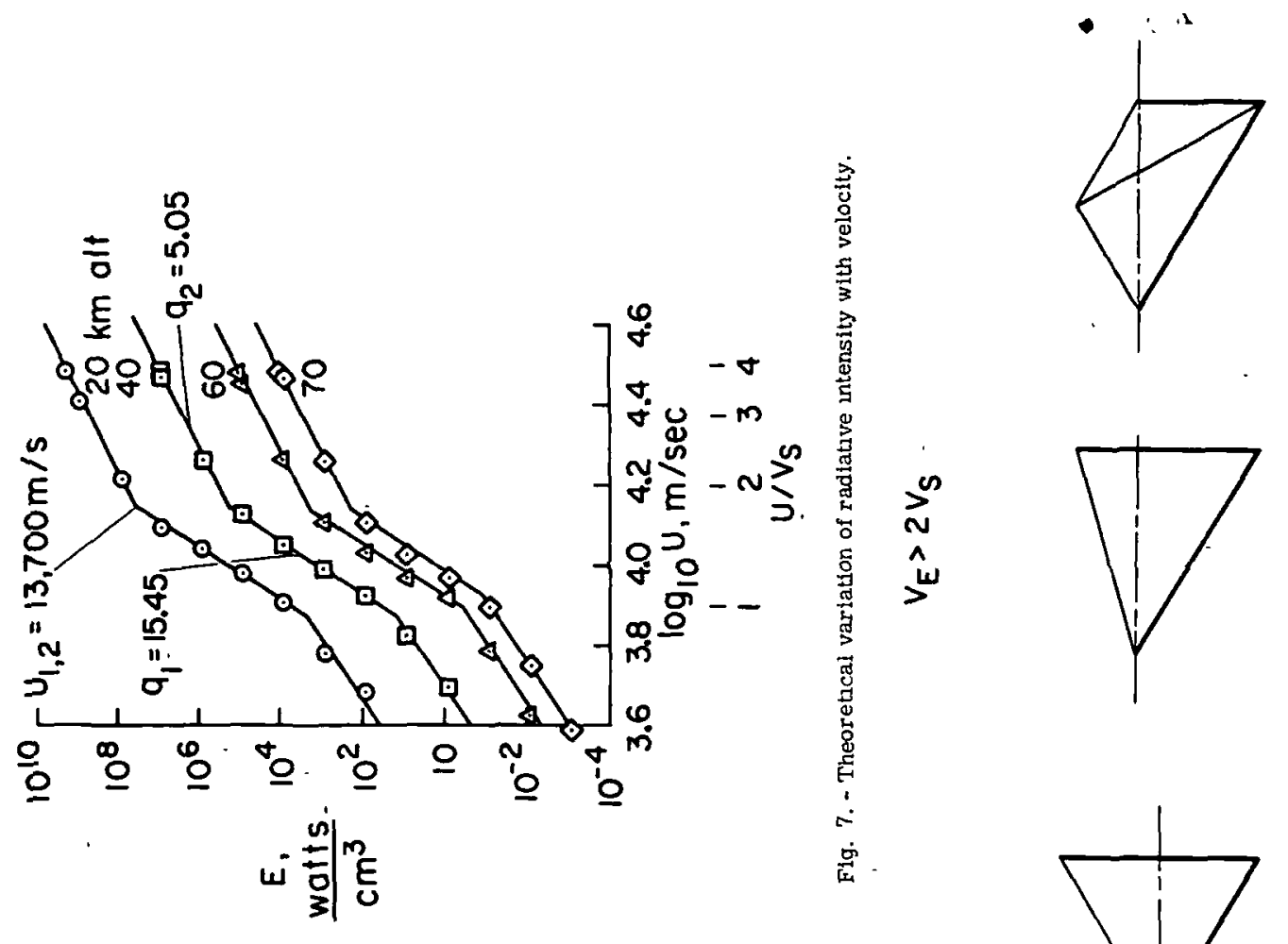

$\frac{0}{2}$
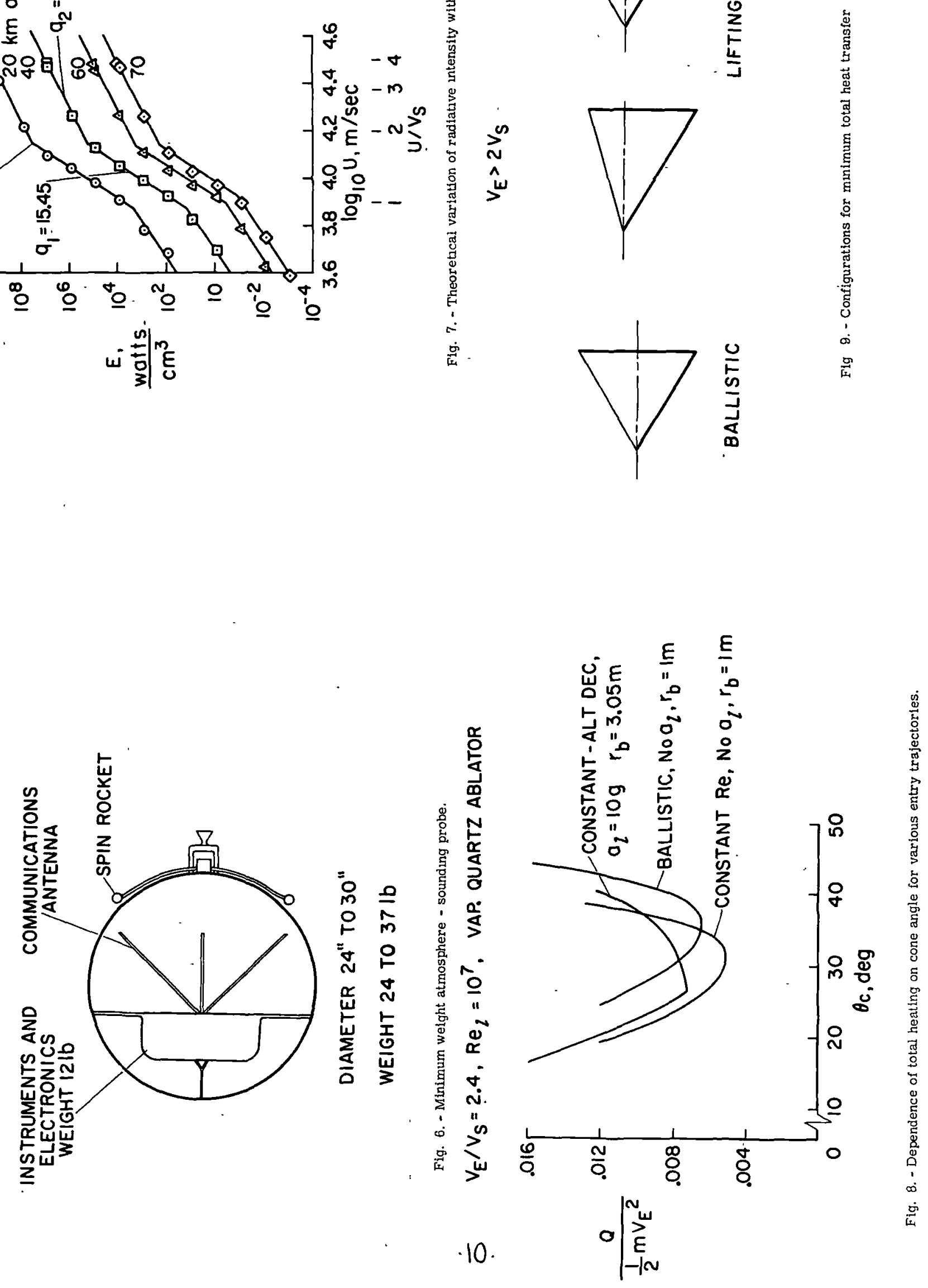


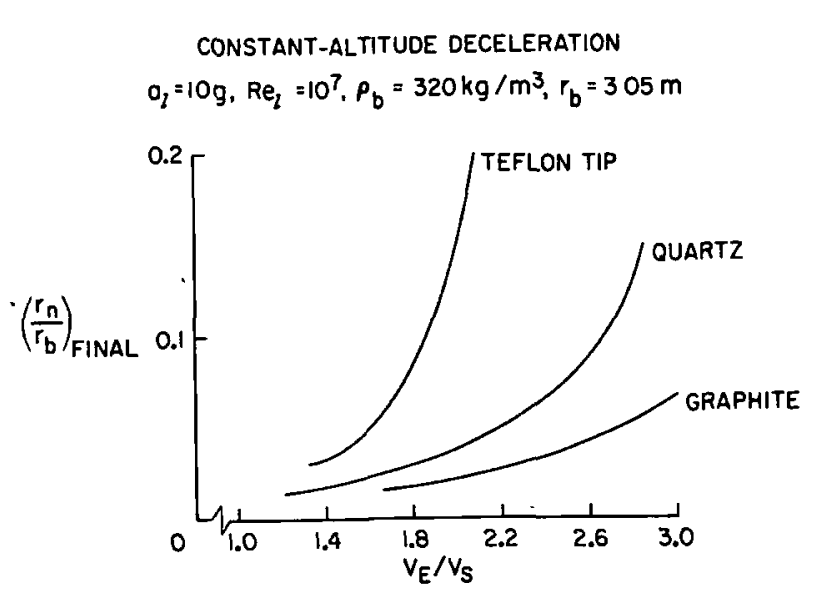

Fig 10 - Theoretscal abiation of contcal tip $v=7 \mathrm{~km} / \mathrm{sec}$

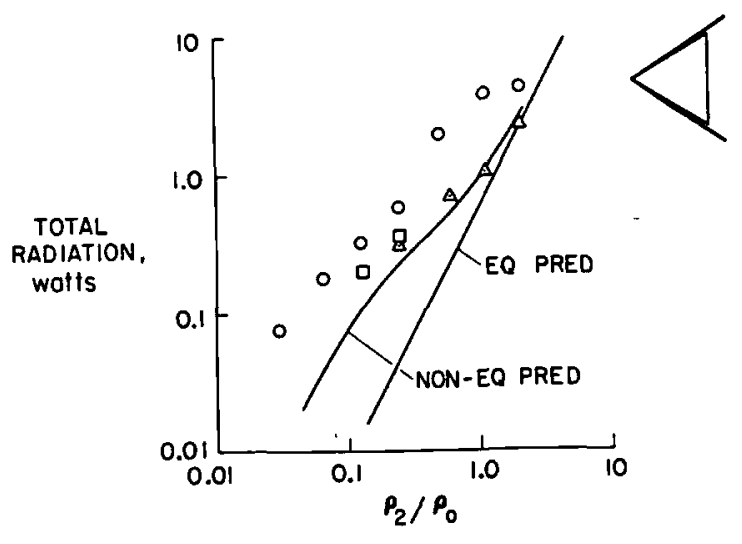

Fig 11 - Ges radiation from $30^{\circ}$ cone,

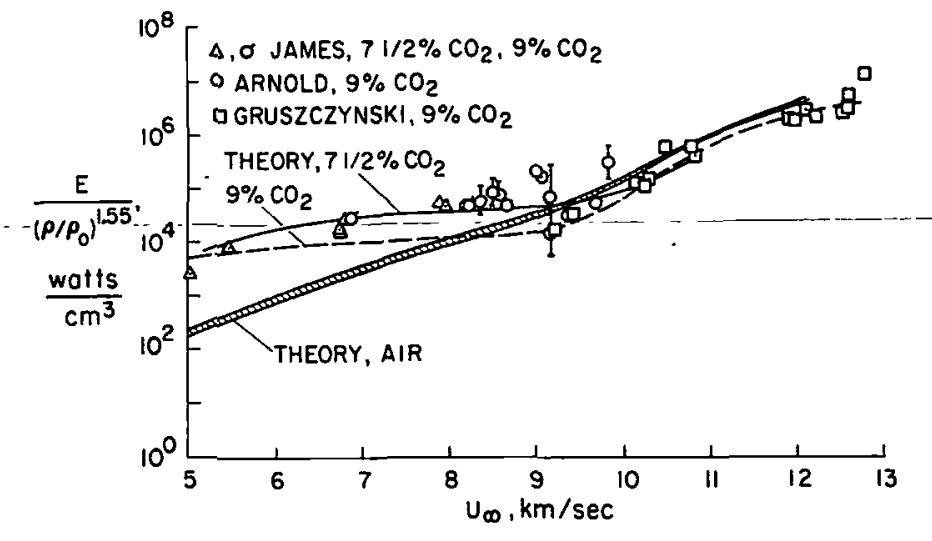

Flg 12 - Speed dependence of $\mathrm{N}_{2}-\mathrm{CO}_{\mathrm{a}}$ shock-layer radiation.

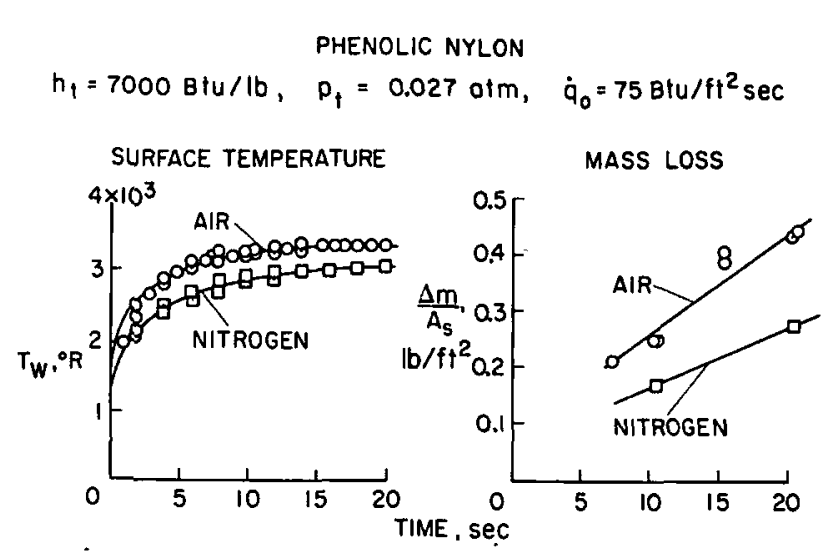

Fig 14 - Effect of chemical reactions on heat-shleld periormance

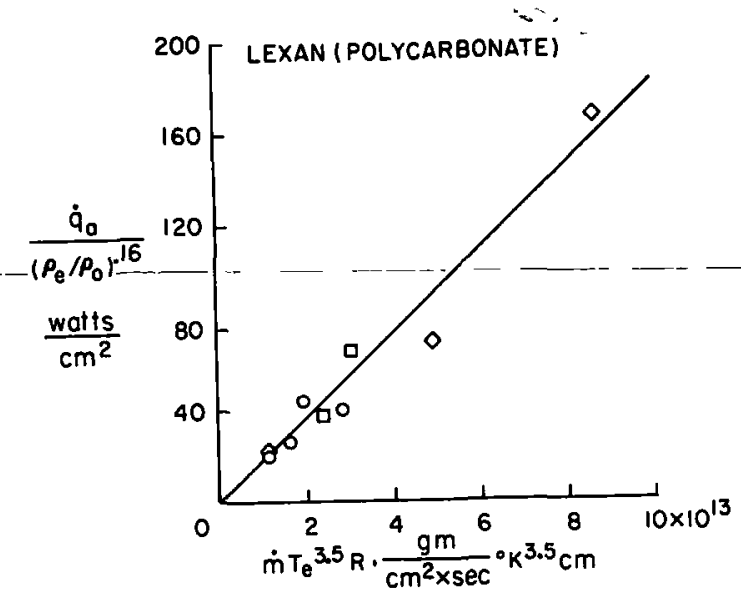

Fig 13 - Radtative heatung from ablation products

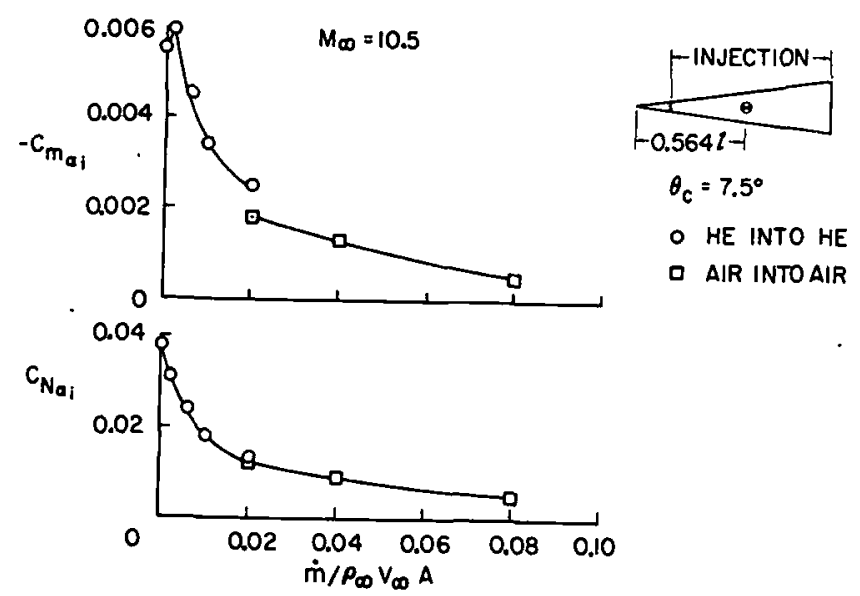

$-11-$ 